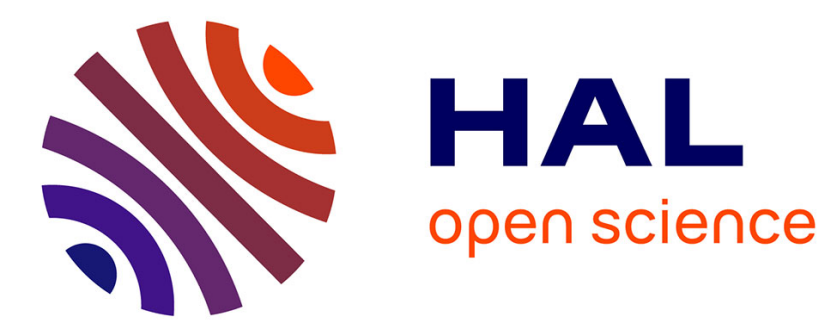

\title{
PROCESSUS EFFECTUAL ET MODĖLE DE PALO ALTO
}

\author{
Fabienne Bornard, Nathalie Goujon, Damien Richard Richard
}

\section{To cite this version:}

Fabienne Bornard, Nathalie Goujon, Damien Richard Richard. PROCESSUS EFFECTUAL ET MODÈLE DE PALO ALTO. Entreprendre \& Innover, 2014, Entreprendre \& Innover, 21-22, pp.30-37. 10.3917/entin.021.0030 . halshs-01233476

\section{HAL Id: halshs-01233476 \\ https://shs.hal.science/halshs-01233476}

Submitted on 29 Nov 2015

HAL is a multi-disciplinary open access archive for the deposit and dissemination of scientific research documents, whether they are published or not. The documents may come from teaching and research institutions in France or abroad, or from public or private research centers.
L'archive ouverte pluridisciplinaire HAL, est destinée au dépôt et à la diffusion de documents scientifiques de niveau recherche, publiés ou non, émanant des établissements d'enseignement et de recherche français ou étrangers, des laboratoires publics ou privés.

\section{(ㄷ)(1) $\$$}

Distributed under a Creative Commons Attribution - NonCommerciall 4.0 International 


\section{PROCESSUS EFFECTUAL ET MODÈLE DE PALO ALTO}

Fabienne Bornard, Nathalie Goujon, Damien Richard

De Boeck Supérieur | «Entreprendre \& Innover »

2014/2 n²1-22 | pages 30 à 37

ISSN 2034-7634

ISBN 9782804188849

Article disponible en ligne à l'adresse :

http://www.cairn.info/revue-entreprendre-et-innover-2014-2-page-30.htm

\section{!Pour citer cet article :}

Fabienne Bornard et al., « Processus effectual et modèle de Palo Alto », Entreprendre \& Innover 2014/2 (n 21-22), p. 30-37.

DOI 10.3917/entin.021.0030

Distribution électronique Cairn.info pour De Boeck Supérieur.

(C) De Boeck Supérieur. Tous droits réservés pour tous pays.

La reproduction ou représentation de cet article, notamment par photocopie, n'est autorisée que dans les limites des conditions générales d'utilisation du site ou, le cas échéant, des conditions générales de la licence souscrite par votre établissement. Toute autre reproduction ou représentation, en tout ou partie, sous quelque forme et de quelque manière que ce soit, est interdite sauf accord préalable et écrit de l'éditeur, en dehors des cas prévus par la législation en vigueur en France. Il est précisé que son stockage dans une base de données est également interdit. 


\title{
Processus effectual et modèle de Palo Alto
}

\author{
> Fabienne Bornard \\ $>$ Nathalie Goujon \\ Damien Richard
}

\section{Résumé}

Les travaux de Sarasvathy sur l'effectuation ont apporté un nouvel éclairage sur l'expertise entrepreneuriale. Cependant ils soulèvent beaucoup de questions, en particulier en situation d'accompagnement. Par exemple, comment aider les entrepreneurs à s'adapter à l'inconfort apparent d'une démarche effectuale ? Partant d'une réflexion interdisciplinaire entre une psychothérapeute praticienne de l'accompagnement et deux enseignants chercheurs, nous étudions le potentiel d'une méthode d’accompagnement fondée sur le modèle de Palo Alto.

\section{Les points forts}

- Comment accompagner les entrepreneurs si l'on ôte le support rassurant d'une approche causale?

- Comment apprendre aux entrepreneurs à s'adapter en souplesse à l'incertitude et aux aléas de l'environnement dans une démarche effectuale, les aider à sortir de certaines routines de pensée et d'action devenues inefficaces, voire nuisibles?

- Sur la base d'une expérience menée au sein d'un incubateur étudiant, nous étudions comment une méthode d'accompagnement fondée sur le modèle de Palo Alto peut répondre à ces questions. 
es composantes de l'expertise entre- preneuriale commencent à être mieux connues, en particulier grâce travaux de Sarasvathy ${ }^{1}$ sur la théorie de l'effectuation. Cependant il reste du chemin à faire pour transposer ces enseignements dans le cadre de l'accompagnement : comment apprendre aux entrepreneurs à s'adapter en souplesse à l'incertitude et aux aléas de l'environnement dans une démarche effectuale ? Comment accompagner les entrepreneurs si l'on ôte le support rassurant d'une approche causale qui guide par étapes vers un objectif ? Un décloisonnement entre la recherche en entrepreneuriat et les pratiques d'accompagnement psychologique peut favoriser l'émergence de nouvelles réponses. En effet, l'approche effectuale vient bousculer des modes de pensée qui nous ont été inculqués, ce qui peut générer une forme de déstabilisation aussi bien du côté des accompagnateurs que des entrepreneurs. Trouver des méthodes permettant d'aider sur le "vif" l'entrepreneur à faire face aux obstacles concrets qu'il rencontre, en lui donnant confiance en ses propres ressources, serait utile.

Cet article présente la méthode dite "de Palo Alto ". Un accompagnement d'entrepreneurs fondé sur ce modèle peut-il constituer un mode d'intervention efficace ? La réflexion proposée est issue d'un dialogue entre une psychothérapeute praticienne de l'accompagnement et deux enseignants chercheurs ayant tous une pratique de l'accompagnement d'entrepreneurs. Notre réflexion est fondée sur des situations réelles d'accompagnement vécues sur une durée de douze mois dans le cadre de l'incubateur de l'EM Lyon, avec deux groupes différents de huit étudiants ayant créé

1 Sarasvathy (Saras), Effectuation, Elements of Entrepreneurial Expertise, Edward Elgar Publishing Limited, 2008. une entreprise. Nous présentons dans un premier temps l'approche de Palo Alto, en particulier la théorie du "but conscient", pour montrer en quoi elle présente une forte connivence avec la théorie de l'effectuation. Puis nous illustrons avec deux cas comment la grille d'analyse de l'école de Palo Alto permet de lever certaines difficultés rencontrées par les entrepreneurs.

Différentes approches théoriques ${ }^{2}$ se rejoignent sur l'idée que l'entrepreneur mène un processus qui repose sur le développement progressif de liens sociaux, impliquant les interlocuteurs du projet, jusqu'à ce qu'ils en deviennent des parties prenantes. Ainsi, petit à petit et avec « les moyens du bord ", le projet se construit.

Ce cheminement génère de nombreuses interactions et émotions, qu'il est parfois difficile de gérer seul. L'entrepreneur doit apprendre à "réguler", c'est-à-dire gérer des interactions avec un environnement mais également gérer ses peurs, colères ou blocages.

Parmi les approches psychologiques, les travaux de Bateson apportent des clés de compréhension sur le fonctionnement humain dans ses interactions. Gregory Bateson (1904-1980), anthropologue et psychologue américain, considère la communication comme la matrice de toutes les activités humaines. Ses recherches sont à l'origine de "l'école de Palo Alto "³ créée au sein du MRI (Mental Research Institute). En 1967, Bateson expose la notion de "but conscient", qui fait écho à Sarasvathy lorsqu'elle pointe les limites du raisonnement causal chez l'entrepreneur.

\footnotetext{
2 Théories du bricolage, de l'effectuation, lean start up... 3 Cf. Wittezaele (Jean-Jacques) et Garcia (Teresa) A la recherche de l'école de Palo Alto, Paris, Seuil, 1992 ou Watzlawick (Paul), et al., Changements, Points Seuil, 1981. (Palo Alto : petite ville près de San Francisco).
} 


\section{Le paradoxe du « but conscient»}

Un " but conscient " implique de penser de manière " linéaire " selon un mode causal : si j'obtiens A au temps 1 , puis $B$ au temps 2, alors j'atteins mon but en $\mathrm{C}$, au temps 3. Il présente également un caractère " vital ": la personne ne peut absolument pas imaginer une autre issue que d'atteindre ce but et de cette manière précise. Ceci l'empêche de percevoir les informations que lui renvoie son environnement. Or, celles-ci auraient pu le conduire à ajuster ses pensées et ses actions. Les "buts conscients " s'avèrent donc des freins à l'atteinte de nos objectifs. Cette idée est parfaitement traduite par le dicton suivant : "le plus sûr moyen d'échouer est de vouloir le succès à tout prix. "4. C'est comme si les buts "décidaient" de ce dont l'individu prend conscience : " si nous laissons nos buts décider seuls de ce qui doit être examiné consciemment, nous n'obtiendrons jamais qu’un sac à malices. " (Bateson, 1967).

Le paradoxe du but conscient tient dans la formule suivante: "Pour atteindre le but, il faut l'abandonner. "Selon Bateson, l'individu doit " juste " s'adapter en souplesse lorsque l'effet émerge.

\section{, La logique effectuale selon}

\section{Sarasvathy}

Les observations de Sarasvathy ${ }^{5}$ mettent fin au mythe de l'entrepreneur visionnaire porteur d'une idée géniale et qui la transforme en projet lucratif. Sarasvathy pointe

4 Ce paradoxe de la rationalité instrumentale a notamment été analysé par le professeur Jon Eslter (Le désintéressement, Seuil, 2009).

5 Voir le numéro 15/2012/ novembre sur le plan d'affaires, dans ce même journal, en particulier l'article de Philippe Silberzahn, pages 9 à 16 . les limites de l'approche dite " causale " de la création d'entreprise qui consiste à raisonner "à rebours » en partant d'un objectif ultime. Comment prendre une décision de fixation d'un prix quand le marché n'existe pas encore ? Sarasvathy a pu observer que les entrepreneurs dits " experts" suivent une logique dite "effectuale " dans laquelle ils partent d'un ensemble de moyens donnés et se concentrent sur la sélection entre les effets possibles. Elle observe que les entrepreneurs experts partent souvent d'une idée parfois floue voire triviale. Ils s'appuient sur ce qui se trouve à leur portée, à savoir, leur personnalité, leur réseau de contacts, et leur savoir. Il existe une forte parenté entre les travaux de Sarasvathy et ceux de Bateson. L'effectuation de Sarasvathy permet au créateur de changer d'objectifs en cours de route, de les construire au fur et à mesure. Elle rejoint le paradoxe du but conscient de Bateson qui décrit comment le fait de se fixer sur un unique but conscient et une démarche linéaire pour l'atteindre, entrave la construction des possibles.

Relisons les trois grands principes de l'effectuation, à la lumière de la théorie du but conscient.

1. "Un tiens vaut mieux que deux tu l'auras" ("The Bird in Hand Principle"): les entrepreneurs experts partent des ressources à leur disposition pour bricoler chemin faisant une stratégie.

2. "La perte acceptable" ("The Affordable Loss Principle ") : L'entrepreneur expert raisonne en perte acceptable. II estime les risques et les coûts et non les gains espérés par l'atteinte d'un but conscient.

3. "Le patchwork fou " ("The Crazy Quilt Principle "): les entrepreneurs à succès 
ont tendance à multiplier les partenariats tous azimuts pour co-construire une offre sur mesure en interagissant avec les parties prenantes (ce que peut empêcher le but conscient).

Comment accompagner des entrepreneurs dans une logique effectuale ? Comment les aider à rester souples et adaptatifs alors même que tout les pousse à rationnaliser, tenter de prévoir et formaliser?

\section{, Le modèle de Palo Alto}

Le modèle de Palo Alto peut répondre en partie à ces questions car il constitue :
- un modèle systémique et interactionnel : l'individu et ses parties prenantes forment un système ayant des interactions ;

- un modèle stratégique (au sens thérapeutique) : il préconise des stratégies d'intervention visant à modifier les comportements interactionnels de l'individu. D’après Wittezaele ${ }^{6}$, " un problème c'est la même difficulté qui revient sans arrêt, car ce que l'on a essayé de faire pour la résoudre la maintient ou la renforce, ainsi elle devient un problème. La cible de notre intervention va être dans un premier temps d'empêcher les personnes de recourir à ces tentatives de solution inefficaces " ;

a été essayé jusque-là sans succès par la personne ou par son entourage pour résoudre le problème?

- Quel est le thème des tentatives de solution?

À un niveau logique supérieur, on cherche à repérer un dénominateur commun aux tentatives de solution. C'est un peu comme si c'était toujours la même musique qui était jouée avec différents instruments. On trouve le thème musical pour changer de morceau et pas seulement d'instrument.

\section{- Quel est donc le thème d'intervention ?}

On cherche alors le thème d'intervention qui se trouve à l'opposé (à $180^{\circ}$ ) de ce que la personne a essayé de faire jusqu'alors. Par exemple affronter sa peur plutôt que de la fuir, répondre au " harceleur " plutôt que de se taire, etc. On a alors un mouvement stratégique qu'il va falloir traduire en action acceptable par la personne.

\section{- Quelle tâche peut-on construire ?}

La tâche est une action recommandée qui va permettre le changement. Les personnes repartent de la séance avec des choses à faire, dire ou penser, qui amèneront un changement par l'arrêt des tentatives de solution ou une action à $180^{\circ}$.

6 Colloque de mars 2006 - " changements de comportements " à Namur (http://www.igb-mri.com). 
- une grille pragmatique de résolution de problèmes : la finalité est non pas la compréhension (pourquoi ?) mais le changement (comment faire autrement ?), la transformation du système par l'action.

L'école de Palo Alto propose une grille d'intervention pragmatique.

Afin d'illustrer cette grille, nous présentons deux cas d'accompagnement d'entrepreneurs. Ces cas ont été simplifiés, les prénoms et situations modifiées dans un souci de pédagogie et de confidentialité.

\section{Vincent : " Réussir à tout prix "}

Vincent, 23 ans, est étudiant en école de commerce. Il a créé son entreprise il y a un an, pendant ses études.
Le principe de cette intervention auprès de Vincent est de lui faire affronter le pire de manière imaginaire, afin de réduire ses angoisses. Regarder les choses en face permet de faire baisser la pression. "Touchez les fantômes et ils disparaissent " disait Paul Watzlawick. Il s'agit donc d'une prescription à l'opposé de ce qu'il tentait de faire en tâchant de ne pas penser à ses peurs. Le fait de se dire "ça va bien se passer " est une tentative de solution inefficace qui ne fait que renforcer la peur.

Ce travail se poursuit par la consigne suivante: prendre, chaque jour, quinze minutes pour affronter le pire de ce qu'on pourrait lui répondre en prospection. Il pourra alors apprivoiser sa peur

\section{Dialogue entre l'accompagnante et Vincent}

- Bonjour Vincent, qu'est-ce qui t'amène? - J'ai perdu la foi et je voulais vous voir, je " pète les plombs". Je n'arrive plus à faire ma prospection téléphonique, ni même à relancer les prospects que j'ai déjà rencontrés, c'est l'horreur!

- D'accord, quand tu te dis que tu vas te faire " jeter " de quoi as-tu le plus peur?

- Peur qu'on me dise que mon positionnement est nul ou qu'on est trop cher.

- C'est déjà arrivé ?

- Non, mais j'ai peur de ça...

- Qu'est-ce qui pourrait se passer de pire?

- Que j'enchaîne les appels téléphoniques et les entretiens et qu'on me dise que mon offre est nulle, qu'on est trop cher...

- Et quoi ? Que tu n’aies plus de clients?

- Oui c'est exactement cela : que nous soyons la risée de tout le monde, qu'on ne fasse plus de chiffre,...

- Et ?...

- Et que nous ne puissions plus nous verser nos SMIC. Que nous devions déposer le bilan et " planter " nos familles et nos amis qui ont mis de l'argent.

- Et encore quoi d'autre... ?

- Et voilà, ce serait l'horreur totale... Ma copine me quitterait ... et mon associé avec son bébé et sa femme seraient à la rue et sans rien...

- Et puis l'ensemble de ta famille te tomberait dessus pour demander des comptes, ta copine aussi en disant que tous les week-ends à bosser où tu la délaissais furent vains et inutiles...

- Oui c'est cela. »

L'accompagnante continue à le questionner car elle sent que tout n'a pas encore été abordé...

« - Et qu'est-ce que tu ferais alors?

- Ben je n'en sais rien, ce serait l'enfer!

- Tu chercherais un autre job ou tu relancerais un nouveau projet ?"

La question le désarçonne.

L'accompagnant: "Mais non, tu serais anéanti et tomberais probablement en dépression... et tu serais obligé de retourner vivre chez tes parents.

- Mais vous êtes atroce de me dire ces trucs...» 
chaque jour. On dit souvent qu'une peur affrontée devient courage alors qu'une peur évitée se transforme en angoisse. Cette technique, appelée "la tâche du pire " peut être utilisée quand le sujet évite sa peur et refuse de l'affronter. Vincent est également reparti avec une question étrange visant à attaquer son but conscient : " Si tu étais sûr que ton entreprise ne marchera jamais, que ferais-tu ?".

Que s'est-il passé pour Vincent ? Le fait d'envisager l'échec comme une possibilité parmi d'autres a apaisé sa peur, il s'est remis en lien avec son intuition et les informations renvoyées par son environnement. Il a arrêté la " prospection dans le dur " pour multiplier les contacts réseau, redirigé à chaque rendez-vous vers des personnes à même d'être intéressées par son offre qu'il a fait évoluer en termes de politique de prix et de positionnement.

Cette action, à l'opposé des efforts précédents, a eu pour effet de le tirer de l'ornière d'une prospection " dans le dur " pour "faire avec qui il est et qui il connaît" (cf Sarasvathy).

\section{Julie : " contrôler l’incontrôlable "}

Julie, 27 ans, est ingénieure et porteuse d'un projet dans les Nouvelles Technologies, dans sa première année de création.

\section{Dialogue au sein du groupe d'entrepre- neurs accompagnés}

- J'ai deux problèmes : d'abord je ne sais pas si je dois lancer mon activité avec 2000 ou 4000 pièces. Si je pars avec un stock trop faible je manquerai des ventes. Si je vois trop grand, j'ai peur de rester avec de la marchandise sur les bras. Je me demande aussi comment connaître les marges de mes fournisseurs pour ne pas me faire avoir. Aujourd'hui, j'y vais au bluffe mais je pense que je devrais avoir une grille précise pour travailler. Cela me rassurerait d'avoir des références de calcul sur chaque catégorie de produits afin d'être sûre de ma négociation. "

L'ensemble du groupe questionne pour mieux comprendre :

"- Mais comment penses-tu que font tes concurrents?

- En fait je n'en sais rien et je n'ai pas vraiment de concurrents pour l'instant.
- Est-ce que c'est déjà arrivé que tes fournisseurs disent non parce que tu exigeais un prix trop faible?

- Non, mais j'ai peur de tomber à côté de la plaque... "

L'accompagnant reprend la parole:

" - Concernant les quantités, personne ne peut savoir mieux que toi combien prévoir. Tu peux à la limite imaginer ce que tu risques de perdre et éventuellement trouver un plan B pour écouler les produits qui te resteront. Comme tout le monde ici, tu navigues à vue. La meilleure décision n'existe pas. Il s'agit de décider et de rendre cette décision bonne en l'assumant. - Mais c'est difficile, j'aimerais bien avoir plus de données pour décider, comme cette grille de prix...

- Je comprends, mais existe-t-il quelque part, quelqu'un qui a cette information? - Non, je ne pense pas car c'est un nouveau marché et je propose aussi une nouvelle manière de travailler... 
En fait, Julie cherche à " contrôler l'incontrôlable ». Elle se sent prise dans une cascade de doutes. Imaginons un arbre des doutes : au départ il y a un tronc, la première question, puis quelques branches, d'autres questions, qui se ramifient en une multitude d'autres branches plus fines... Julie se perd dans ses ramifications où aucune réponse n'existe et vit un enfer psychologique.

L'intervention consiste à lui renvoyer la question : est-ce que quelqu'un dans l'univers a une réponse ? Si la réponse est " oui ", alors il faut la chercher. En revanche, si la réponse est " non ", alors il faut " couper au niveau du tronc l'arbre des doutes $"{ }^{7}$, c'est-à-dire évacuer la première question à laquelle personne n'a de réponse. Le risque est, sinon, d'éviter la prise de décision, ce qui peut conduire à bloquer l'entreprise.

Cette intervention est utilisée dans les situations de doute pathologique et vise à arrêter les tentatives de solutions dont le thème est : " chercher des réponses qui n'existent pas ". Il s'agit là d'un recadrage. Ce cas illustre le caractère parfois inopérant d'une approche causale linéaire. l'intervention a permis de renouer avec une approche effectuale.

\section{Cycle d'expansion des ressources}

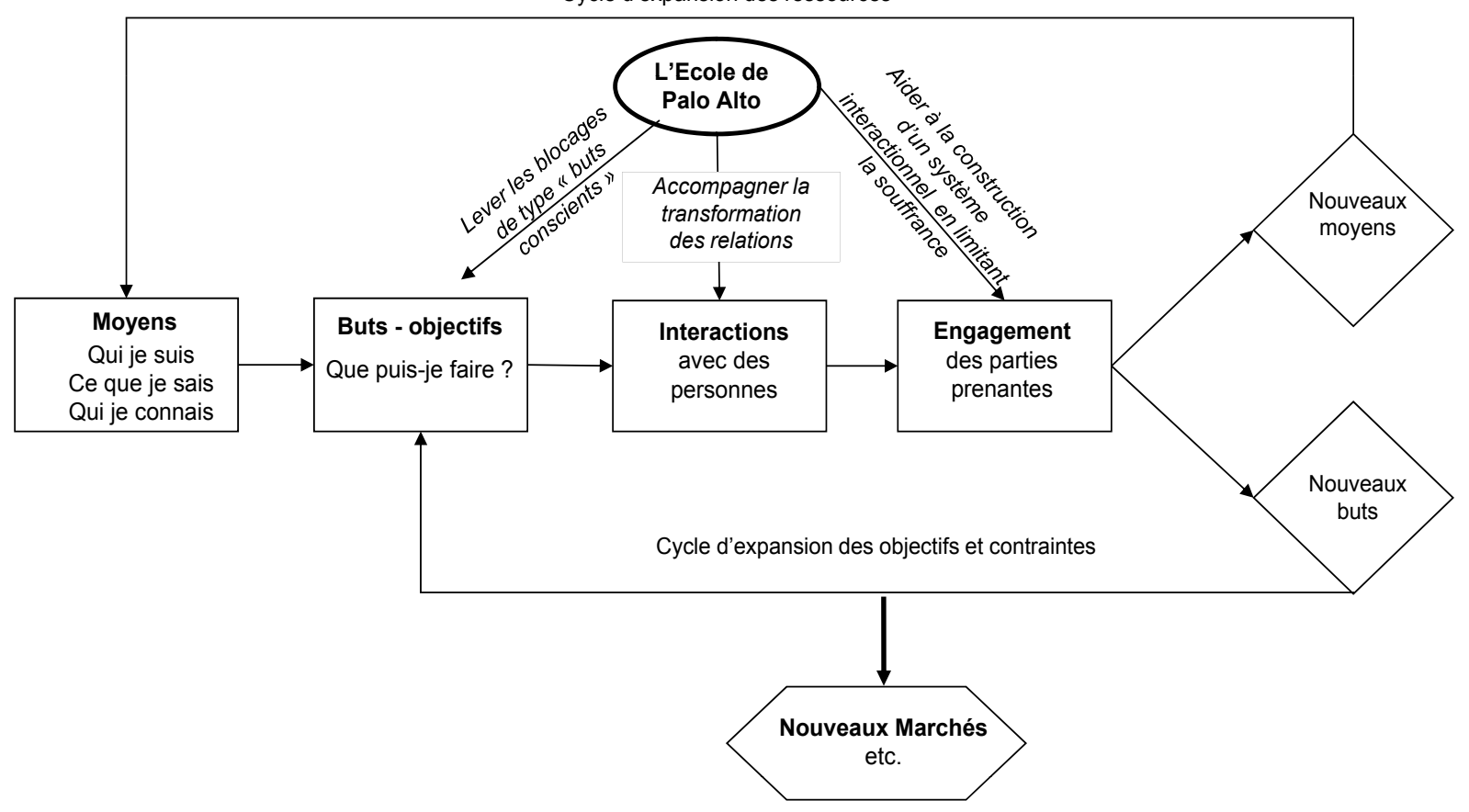

L'apport de Palo Alto au processus effectual

Source : Stuart Read, Sarasvathy, et al. 2010

7 Cf. Nardone (Giorgio) et Watzlawick (Paul), L'art du changement, L'esprit du Temps, 2003. 


\section{Faire évoluer}

\section{l'accompagnement}

Cette discussion chercheur/praticien propose quelques illustrations de l'accompagnement avec le modèle de Palo Alto qui présente un double avantage. Ce modèle permet à l'entrepreneur d'imaginer et de tester de nouvelles solutions pour gagner en souplesse, afin de mieux vivre une démarche effectuale. De plus, il invite à remettre en question ses représentations et routines d'action pour mieux s'adapter aux aléas de l'environnement.

Mais son utilisation dans l'accompagnement des entrepreneurs présente aussi certaines limites. L'intervention n'est possible que si le problème est abordé par la personne et si elle souhaite un changement. De plus, l'intervention n'est pas simple. Le praticien doit définir le problème, identifier les tentatives de solution et leur thème, élaborer une stratégie de changement parfois déstabilisante et convaincre la personne de réaliser les tâches prescrites.

Lorsqu'elle est mise en œuvre auprès de porteurs de projet cette méthode permet de donner de la souplesse aux interactions avec les parties prenantes. Ce modèle pragmatique visant à identifier les tentatives de solution et les buts conscients permet d'élaborer, dans la discussion, une stratégie plus économe en temps et en souffrance. Il permet d'accompagner le processus effectual : laisser le projet se construire " naturellement " sans chercher à suivre un chemin linéaire et laisser l'entrepreneur faire avec "qui il est », avec " ce qu'il sait " et avec " qui il connaît ". L'horizon est de renouer avec une vision optimiste en accédant à la liberté et à la responsabilité d'écrire soi-même son propre destin en tant qu'entrepreneur.

\section{Trois références pour en savoir plus}

(1) À la recherche de l'école de Palo Alto de I.-J. Wittezaele et T. Garcia, chez Seuil, CouleurPsy, 2006.

(2) Vers une écologie de l'esprit (2 tomes), de G. Bateson, chez Editions du Seuil, Points Essais, 1980.

(3) Effectuation : les principes de l'entrepreneuriat pour tous, de Philippe Silberzahn, Pearson, 2014.

Fabienne Bornard est enseignant chercheur à I'INSEEC Alpes-Savoie, spécialisée en entrepreneuriat et stratégie, après dix années d'expérience dans le conseil et la formation auprès des entreprises. Ses axes de recherches concernent la façon dont les entrepreneurs pensent et prennent des décisions, dont sa thèse sur leurs représentations mentales, et comment les former et les accompagner.

Nathalie Goujon est coach et formatrice en entreprises et chargée de cours au sein d'écoles de commerce (EM Lyon, GEM, ESDES, INSEEC AlpesHaute savoie). Elle a une expérience en entreprise d'une dizaine d'années, dans des grands groupes internationaux comme des PME industrielles.

Damien Richard est enseignant-chercheur à I'INSEEC Alpes-Savoie. Ses travaux portent sur le bienêtre au travail à travers les espaces de discussion et l'accompagnement de dirigeants et d'équipes. Il fait partie de la Chaire Management et Santé au travail (CERAG-IAE Grenoble). 